Internat. J. Math. \& Math. Sci.

Vol. 24, No. 12 (2000) 821-823

S0161171200002702

(C) Hindawi Publishing Corp.

\title{
ON A FOUR-GENERATOR COXETER GROUP
}

\author{
MUHAMMAD A. ALBAR
}

(Received 4 December 1999)

ABSTRACT. We study one of the 4-generator Coxeter groups and show that it is SQ-universal (SQU). We also study some other properties of the group.

Keywords and phrases. Group presentation, Reidemeister-Schreier process, Coxeter group, SQ-universality.

2000 Mathematics Subject Classification. Primary 20 F05.

1. Introduction. We consider the Coxeter group $P$ given by the presentation

$$
\begin{aligned}
P= & \left\langle x_{1}, x_{2}, x_{3}, x_{4}\right| x_{1}^{2}=x_{2}^{2}=x_{3}^{2}=x_{4}^{2}=\left(x_{1} x_{2}\right)^{3} \\
& \left.=\left(x_{2} x_{3}\right)^{3}=\left(x_{1} x_{3}\right)^{3}=\left(x_{1}, x_{4}\right)^{3}=\left(x_{3} x_{4}\right)^{3}=\left(x_{2} x_{3}\right)^{3}=e\right\rangle .
\end{aligned}
$$

The Coxeter graph of this group is clearly just a combinatorial tetrahedron:

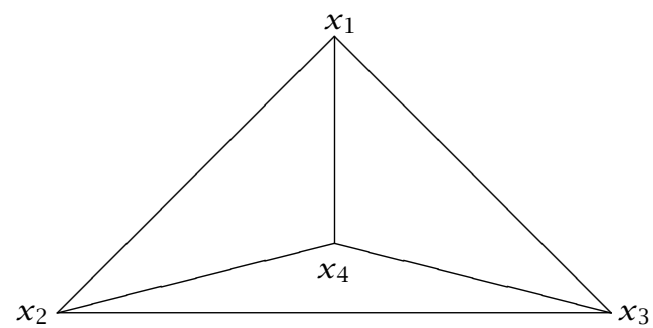

We observe that each face is the graph of the Euclidean triangle group $\triangle(3,3,3)$ which is an affine Weyl group and this contains a nilpotent subgroup of finite index. The group $P$ is infinite and it will be interesting to see its largeness by answering whether it is SQ-universal or not.

2. SQ-universality. We let $S_{3}$ be the symmetric group of degree 3 . Thus

$$
S_{3}=\left\langle y_{1}, y_{2} \mid y_{1}^{2}=y_{2}^{2}=\left(y_{1} y_{2}\right)^{3}=e\right\rangle .
$$

We consider the map $\theta: P \rightarrow S_{3}$ defined by

$$
\theta\left(x_{1}\right)=y_{1}, \quad \theta\left(x_{2}\right)=y_{2}, \quad \theta\left(x_{3}\right)=\theta\left(x_{4}\right)=y_{1} y_{2} y_{1}
$$

It is easy to see that $\theta$ is an epimorphism and $P / \operatorname{ker} \theta \cong S_{3}$. A Schreier transversal for $S_{3}$ in $P$ is $\left\{e, x_{1}, x_{2}, x_{1} x_{2}, x_{2} x_{1}, x_{1} x_{2} x_{1}\right\}$. A straightforward application of the 
Reidemeister-Schreier process gives the following presentation for $\operatorname{ker} \theta$ :

$$
\operatorname{ker} \theta=\left\langle a, b, c, d \mid(a d)^{3}=(b c)^{3}=(a b c d)^{3}=e\right\rangle .
$$

Letting $a=d^{-1}$ and $b=c^{-1}$, we see that $\operatorname{ker} \theta$ is mapped homomorphically onto the free group of rank $2, F_{2}$. Hence $\operatorname{ker} \theta$ is SQU. Since the index of $\operatorname{ker} \theta$ in $P$ is finite (6), we get that $P$ is also SQU [4].

3. The growth series. Let $(P, X)$ be a Coxeter system and let $Y \subseteq X$. We denote the subgroup of $P$, generated by $Y$, by $P_{Y}$. Then $\left(W_{Y}, Y\right)$ is also a Coxeter system. In Bourbaki [2, Section 1 of Chapter 4], Exercise 26 gives the following formula for computing the growth series of $P$ (word growth in the sense of Milner and Gromov):

$$
\sum_{Y \subseteq X} \frac{(-1)^{|y|}}{P_{Y}(t)}= \begin{cases}\frac{t^{m}}{P(t)} & \text { if } P \text { is finite, } \\ 0 & \text { if } P \text { is infinite. }\end{cases}
$$

In the formula, $G(t)$ is the growth series of $G, m$ is the length of the unique element of $P$ of maximal length.

We use (3.1) to compute $P(t)$. We compute $P(t)$ in steps corresponding to the cardinality of $Y$ :

$|Y|=0$ is the trivial subgroup with growth series $\gamma_{0}=1$.

$|Y|=1$ four cyclic subgroups of order 2 with growth series $\gamma_{1}=1+t$.

$|Y|=2$ six dihedral subgroups of order 6 with growth series $\gamma_{2}=(1+t)\left(1+t+t^{2}\right)$.

$|Y|=3$ four affine subgroups with growth series given by $1 / \gamma_{0}-3 / \gamma_{1}+3 / \gamma_{2}-$ $1 / \gamma_{3}=0$, that is, $\gamma_{3}=\left(1+t+t^{2}\right) /(1-t)^{2}$.

$|Y|=4$ the whole group with growth $\gamma_{4}(t)=P(t)$ given by $1 / \gamma_{0}-4 / \gamma_{1}+6 / \gamma_{2}-$ $4 / \gamma_{3}+1 / \gamma_{4}=0$, that is, $\gamma_{4}=(1+t)\left(1+t+t^{2}\right) /(1-t)\left(1-t-3 t^{2}\right)$.

The growth coefficients $\left\{c_{n}\right\}$ are given by the linear recurrence $c_{0}=1, c_{1}=4, c_{2}=$ $12, c_{3}=30, c_{n}=2 c_{n-1}+2 c_{n-2}-3 c_{n-3}, n \geq 4$ (see [3]). We observe from the growth series $\gamma_{4}$ that zeros of the denominator are not on the unit circle. This implies that $P$ has no nilpotent subgroup of finite index-in accordance with the fact that $P$ is SQU.

It is possible to show that the group $P$ and the Geisking group $G=\langle x, y| x^{2} y^{2}=$ $x y>$ are isometric and hence $\gamma_{4}$ is also the growth series of $G$ (see [3]). In [1], it appears that the two Coxeter groups $T_{n}$ and $S_{n}$ are also isometric and so have the same growth series.

4. The commutator subgroup. Using the Reidemeister-Schreier process, we get the following presentation for $P^{\prime}$ :

$$
P^{\prime}=\left\langle x, y, z \mid x^{3}=y^{3}=z^{3}=(x y)^{3}=(x z)^{3}=\left(y z^{-1}\right)^{3}=e\right\rangle .
$$

We use $P^{\prime}$ to show that $P$ is SQU in a different method. Let $\mathrm{K}$ be the normal closure of the elements $x y^{-1}, x z^{-1}, y z^{-1}$ in $P^{\prime}$. The group $K$ has index 3 in $P^{\prime}$. Using the Reidemeister-Schreier process, we get the following presentation for $K$ :

$$
\begin{gathered}
K=\left\langle u_{1}, u_{2}, u_{3}, v_{1}, v_{2}, v_{3}\right| v_{1}^{2}=v_{2}^{2}=v_{3}^{2}=u_{1} u_{2} u_{3} \\
\left.=u_{1} u_{3} u_{2}=v_{1} v_{2} v_{3}=u_{1} v_{2} u_{3} v_{1} u_{2} u_{3}=e\right\rangle .
\end{gathered}
$$


Letting $u_{3}=v_{3}=e$, we see that $K$ is mapped homomorphically onto $Z * Z_{3}$. Since $Z * Z_{3}$ is SQU (see [4]), therefore $K$ is SQU. Since $K$ is of finite index in $P^{\prime}$ and $P^{\prime}$ is of finite index in $P$, we get that $P$ is SQU.

Acknowledgement. The author thanks King Fahd University of Petroleum and Minerals for supporting him in his research.

\section{REFERENCES}

[1] M. A. Albar, M. A. Al-Hamed, and N. A. Al-Saleh, The growth of Coxeter groups, Math. Japon. 47 (1998), no. 3, 417-428. MR 99f:20066. Zbl 912.20032.

[2] N. Bourbaki, Éléments de Mathématique. Fasc. XXXIV. Groupes et Algèbres de Lie. Chapitre IV: Groupes de Coxeter et Systèmes de Tits. Chapitre V: Groupes Engendrés par des Réflexions. Chapitre V: Systèmes de Racines, Actualités Scientifiques et Industrielles, no. 1337, Hermann, Paris, 1968, 288 pp. MR 39\#1590. Zbl 186.33001.

[3] D. L. Johnson and H.-J. Song, The growth series of the Gieseking group, Discrete Groups and Geometry (Birmingham, 1991), pp. 120-124, London Math. Soc. Lecture Note Ser., no. 173, Cambridge Univ. Press, Cambridge, 1992. MR 93m:20049. Zbl 769.57003.

[4] P. M. Neumann, The SQ-universality of some finitely presented groups. Collection of articles dedicated to the memory of Hanna Neumann, I., J. Austral. Math. Soc. 16 (1973), 1-6. MR 48\#11342. Zbl 267.20026.

Muhammad A. Albar: Department of Mathematical Sciences, King Fahd University of Petroleum ANd Minerals, Dhahran 31261, SAUdi ARabia 


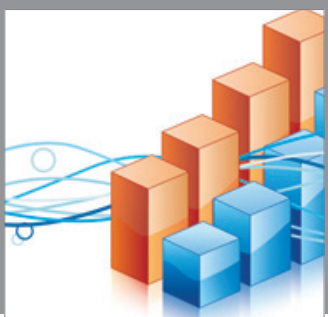

Advances in

Operations Research

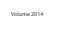

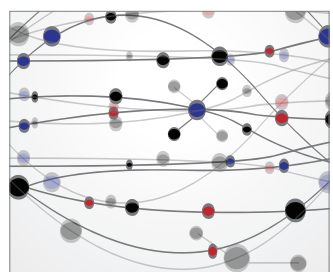

\section{The Scientific} World Journal
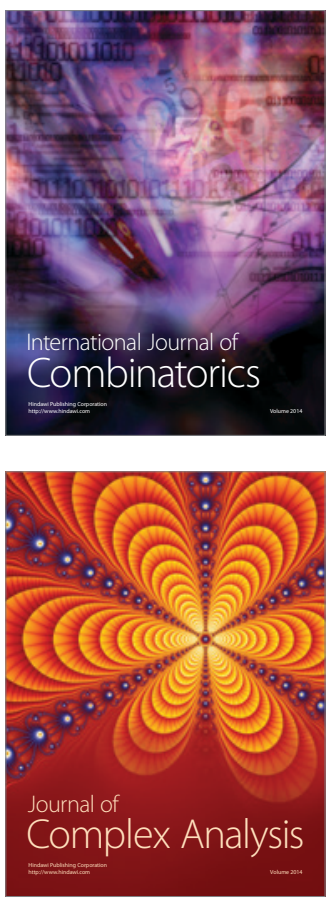

International Journal of

Mathematics and

Mathematical

Sciences
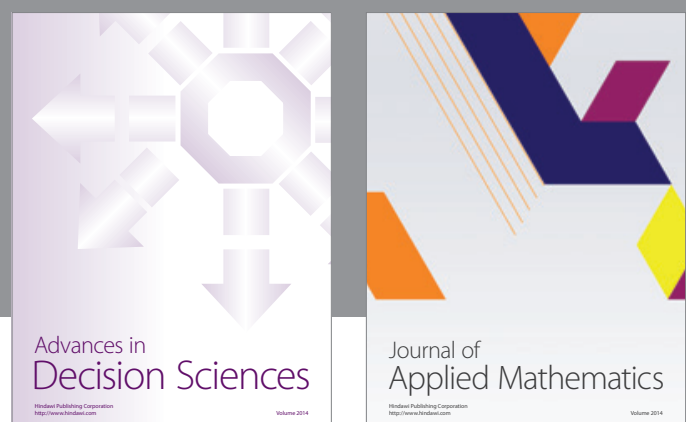

Journal of

Applied Mathematics
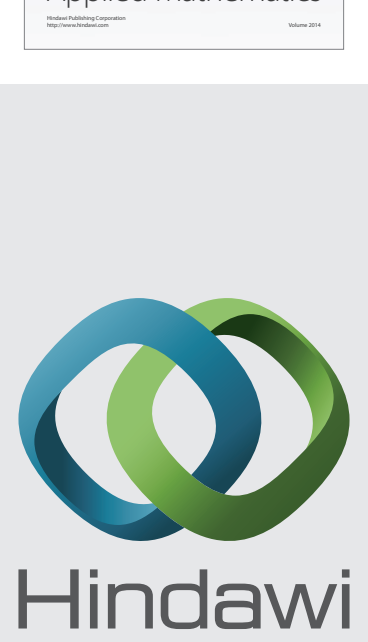

Submit your manuscripts at http://www.hindawi.com
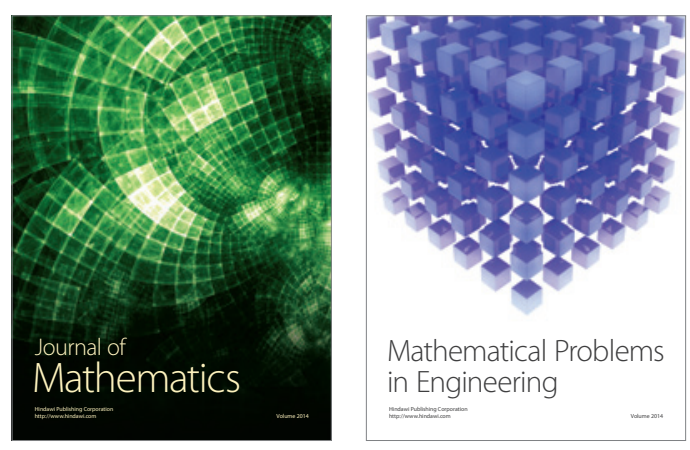

Mathematical Problems in Engineering
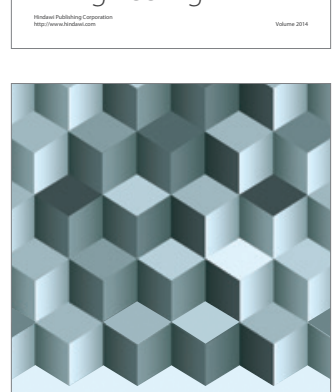

Journal of

Function Spaces
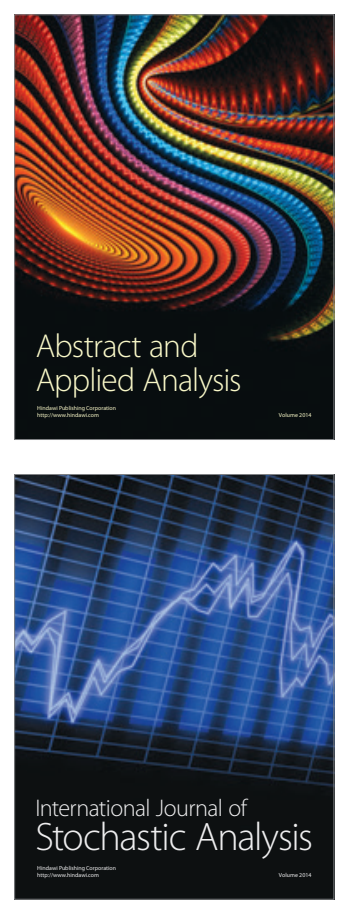

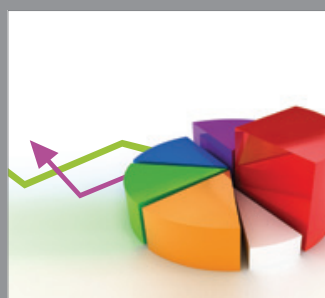

ournal of

Probability and Statistics

Promensencen
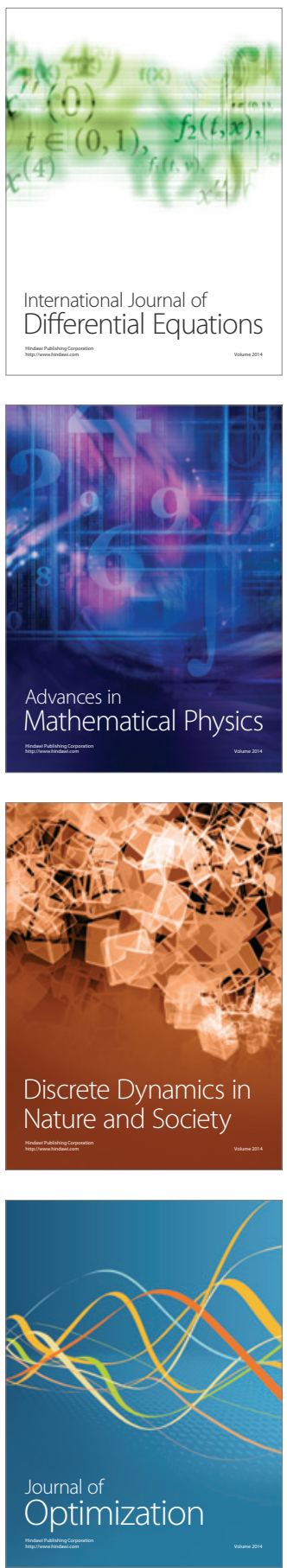